\title{
ICRH ANTENNA DESIGN AND MATCHING
}

\author{
P. Dumortier, A.M. Messiaen \\ Laboratory for Plasma Physics -Ecole Royale Militaire/Koninklijke Militaire School \\ EURATOM-Belgian State Association, Trilateral Euregio Cluster \\ avenue de la Renaissance 30, B-1000 Brussels, Belgium \\ Tel.: (32 2) 742 65 77, Fax.: (32 2)735 2421, e-mail: pierre.dumortier@rma.ac.be
}

\begin{abstract}
$\mathrm{RF}$ heating and current drive in the ion cyclotron frequency range is widely used on existing machines and is planned on ITER. After a brief reminder of transmission line theory concepts the paper illustrates the process of antenna design by explaining some key design choices made for the ITER ICRH antenna. Finally the most common matching schemes are reviewed.
\end{abstract}

\section{INTRODUCTION}

The principle of heating and current drive by plasma waves has been addressed in several other lectures $[1,2,3,4]$. In particular it has been seen [2] that the antenna current strap can be described as a stripline with equivalent transmission line distributed parameters. The matching circuit is composed of transmission line components and hence its response can also be described by transmission line theory. Basic transmission line concepts are therefore reminded before going to antenna design and matching.

It is important to note that the time dependence of the various quantities is in $e^{+i \omega t}$ in this lecture, which is the "engineering" transmission line convention whereas the "physical" convention generally used to describe the wave propagation is time dependence in $e^{-i \omega t}$. As the $e^{+i \omega t}$ factor appears on all timedependent terms it is customary to suppress it on all terms in the different expressions.

\section{TRANSMISSION LINE MODELING}

Transmission line theory can be approached either from an extension of circuit theory or from a specialization of the Maxwell's equations. The first approach is favored here. The main difference with circuit theory lays in the physical size of the electrical circuit: the transmission line size is of the order of magnitude of the wavelength whereas it is much smaller in circuit theory. A transmission line is a distributed parameter network along which voltage and current vary in amplitude and phase. Excellent overview of transmission line theory can be found in $[5,6]$.

A. The telegraphers equations

The voltage $V$ and current $I$ on the line vary due to the presence of the following distributed parameters:

- the series impedance per unit length $Z=R+$ $i X=R+i \omega L(\Omega / m)$, where $R(\Omega / m)$ is the series resistance per unit length and accounts for the conductor losses, $X(\Omega / m)$ and $L(H / m)$ are the series reactance and inductance per unit length.

- the shunt admittance per unit length $Y=G+$ $i B=G+i \omega C(S / m)$, where $G(S / m)$ is the shunt conductance per unit length and accounts for the dielectric losses, $B(S / m)$ and $C(F / m)$ are the shunt susceptance and capacitance per unit length.

The evolution of the voltage and current along the line is described by two second-order differential equations known as the telegraphers' equations:

$$
\frac{d^{2} V}{d z^{2}}=-Z Y V ; \frac{d^{2} I}{d z^{2}}=-Z Y I
$$

The solutions to these equations are the superposition of two traveling waves:

$$
\begin{gathered}
V(z)=V_{0}^{+} e^{-\gamma z}+V_{0}^{-} e^{+\gamma z}=V^{+}+V^{-} \\
I(z)=\frac{1}{Z_{0}}\left(V_{0}^{+} e^{-\gamma z}-V_{0}^{-} e^{+\gamma z}\right)=I^{+}+I^{-}
\end{gathered}
$$

where the $e^{-\gamma z}$ term represents a wave propagating in the positive $\mathrm{z}$ direction and the term $e^{+\gamma z}$ represents a wave traveling in the negative $z$ direction.

The time dependent solution for the voltage (it has a similar form for the current) is:

$$
V(z, t)=V_{0}^{+} e^{-\alpha z} e^{i(\omega t-\beta z)}+V_{0}^{-} e^{+\alpha z} e^{i(\omega t+\beta z)}
$$

The complex propagation constant is :

$$
\gamma=\alpha+i \beta=\sqrt{Z Y}=\sqrt{(R+i \omega L)(G+i \omega C)},
$$


where $\alpha$ is the attenuation constant and $\beta$ is the phase constant or wave number; $\alpha=0$ for a lossless line.

$$
Z_{0}=\sqrt{\frac{Z}{Y}}=\sqrt{\frac{R+i \omega L}{G+i \omega C}}
$$

is the characteristic impedance of the line. Note that $\gamma$ and $Z_{0}$ are functions of the frequency.

\section{B. Transfer matrix and $\mathrm{Z}$ transformation}

If we know the voltage $V_{\text {out }}$ and current $I_{\text {out }}$ at one point in the line, chosen to be at $z=0$, Eqs. 2 and 3 allow us to determine the constants $V_{0}^{+}$and $V_{0}^{-}$. Knowing the characteristics of the line $(\gamma$ and $Z_{0}$ ) and making use of Eqs. 2 and 3 we relate the voltage $V_{\text {in }}$ and current $I_{\text {in }}$ in $z=-l$ to $V_{\text {out }}$ and $I_{\text {out }}$ in $z=0$ by the transfer matrix:

$$
\left(\begin{array}{l}
V_{\text {in }} \\
I_{\text {in }}
\end{array}\right)=\left(\begin{array}{cc}
\cosh (\gamma l) & Z_{0} \sinh (\gamma l) \\
\frac{\sinh (\gamma l)}{Z_{0}} & \cosh (\gamma l)
\end{array}\right)\left(\begin{array}{l}
V_{\text {out }} \\
I_{\text {out }}
\end{array}\right)
$$

Also, the impedance at any point $(z=-l)$, defined as the ratio of the voltage to the current at this point, is defined if we know the impedance at another point $(z=0)$ :

$$
Z_{\text {in }}=Z_{0} \frac{Z_{\text {out }}+Z_{0} \tanh (\gamma l)}{Z_{0}+Z_{\text {out }} \tanh (\gamma l)}
$$

\section{Reflection coefficient and VSWR}

The (voltage) reflection coefficient at one point in the line $(z=-l)$ is expressed as the ratio of the reflected voltage to the incident voltage at this point and is linked to the reflection coefficient at another point in the line $(z=0)$ by:

$$
\Gamma=\frac{V^{-}}{V^{+}}=\Gamma_{0} e^{-2 \gamma l}
$$

Note the factor 2 appearing in the exponential term. It comes from the fact that the combined wave is the superposition of two waves, the incident and reflected waves, traveling in opposite direction at the same phase velocity.

The reflection coefficient at any point is linked to the impedance at the same point by:

$$
\Gamma=\frac{Z-Z_{0}}{Z+Z_{0}}
$$

The Voltage Standing Wave Ratio (or VSWR) is defined as the ratio of the amplitudes of the maximum and of the minimum voltages at a given point and is linked to the modulus of the reflection coefficient at this point by:

$$
S=\frac{V_{\max }}{V_{\min }}=\frac{1+|\Gamma|}{1-|\Gamma|}
$$

For a matched line $|\Gamma|=0$ and $S=1$.

\section{ICRH ANTENNA DESIGN}

\section{A. Antenna components}

An example of ICRH antenna is shown in figure 1. It is one of the two 2-straps antennas of TEXTOR, where each antenna is powered by a $2 \mathrm{MW}$ generator. The current strap is generally a strip-line that

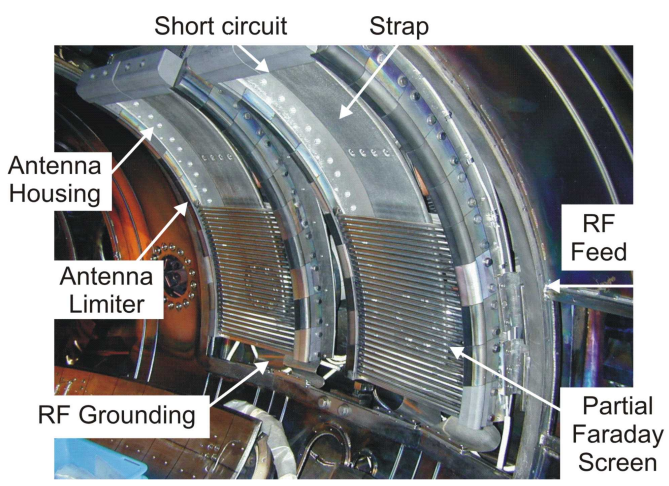

Figure 1: Two-strap TEXTOR ICRH antenna.

is short-circuited at one end to produce the magnetic field exciting the fast magnetosonic wave $[2,3]$. Most recent antennas are arrays of poloidally and toroidally spaced straps. The phase and amplitude of the currents flowing in the different straps of the array need to be controlled to excite the required wave spectrum corresponding to the requested physics scenario (see last section on matching).

The strap is enclosed in the antenna box, part of the antenna housing, which is protected from the plasma by side limiters. It is not only a mechanical structure onto which the current straps, the screen bars and the feeding transmission lines are mounted but it also acts as a return conductor for the RF currents.

The geometry of the straps and antenna box will be subject to an optimization, constrained to the available physical space, to maximize the coupling while remaining below the current, voltage and electric field limits.

The antenna is generally mounted on the outboard equatorial plane of the machine. It is either mounted in the gap between the vessel and the plasma (TEXTOR, ASDEX-U, JET) or built as a whole assembly inside a port plug that is mounted onto the vessel (Tore-Supra, JT-60U, ITER). The first option requires smaller openings in the vessel as the components can be brought inside the machine by an access hole and possibly allows the deployment of larger radiating areas but the available depth can be reduced, impacting on the coupling. The advantages of the plug antenna are easier maintenance and remote handling. It generally has smaller radiating areas, partly compensated by the larger available depth.

$\mathrm{RF}$ grounding of the antenna avoids the excitation of unwanted modes (e.g. in the cavity formed by 
the liner and the vessel on TEXTOR).

A Faraday shield or screen consisting in a slotted frame or a series of bars is generally placed in front of the straps. Its main role is to filter out the electric field parallel to the static magnetic field. For this reason it is preferably oriented parallel to the static magnetic field (what will only occur for a chosen rotational transform).

The antenna is fed by Vacuum Transmission Lines (or VTLs). They connect the antenna inside the machine to the pressurized transmission lines outside the machine and may contain some pre-matching elements.

The vacuum windows or vacuum feedthroughs are critical elements of the ICRH systems. Their primary goal is to enter the RF into the vacuum vessel without breaking the vacuum. They see the machine vacuum on the antenna side and either a private vacuum or pressurized lines on the generator side. They also act as a mechanical support for the inner conductors of the feeding lines and as tritium barriers, when necessary. As they are generally located in sections with high VSWR they must be able to cope with high voltages, of the order of $40-50 \mathrm{kV}$. They generally are constituted of cylindrical or conical ceramics (usually $\mathrm{Al}_{2} \mathrm{O}_{3}$ ) brazed onto the inner and outer metallic conductors of the cylindrical coaxial transmission line. ICRH technology is further discussed in [7].

B. Key design choices for the ITER ICRH antenna

\section{The ITER ICRF antenna [8, 9]}

The main functional requirements for the ITER ICRH system are heating, current drive and wall conditioning. The key specifications are the following:

- Nominal power per antenna (2 antennae are foreseen at the moment): $20 M W$;

- Frequency range: $40-55 \mathrm{MHz}$;

- Quasi-CW operation;

- Phased antenna array (6 poloidal by 4 toroidal array of straps);

- Location: equatorial port plug.

The control of the currents (amplitude and phase) in the different columns of straps combined to the broad frequency range allows covering all requested physics scenarios to fulfill the functional specifications.

The ITER ICRH port plug (figure 2) contains $4 \mathrm{RF}$ modules and is cantilevered from the port plug flange, where it is connected to the vacuum vessel. Its inside acts as a neutron shield, reducing the level of radiation at the back flange below a level permitting hands-on operation after some time for maintenance. The port plug is put in place by remote handling.

One RF module consists of two columns of three short current straps. Each strap is connected to a $15 \Omega$ characteristic impedance cylindrical coaxial feeder. Three poloidal straps with their feeders are connected in parallel by a 4-port junction (4PJ) to form a triplet of straps. The $4 \mathrm{PJ}$ is connected to one $20 \Omega$ characteristic impedance RF feeding line containing a vacuum window acting as a mechanical support for the inner conductors, a vacuum and a tritium barrier.

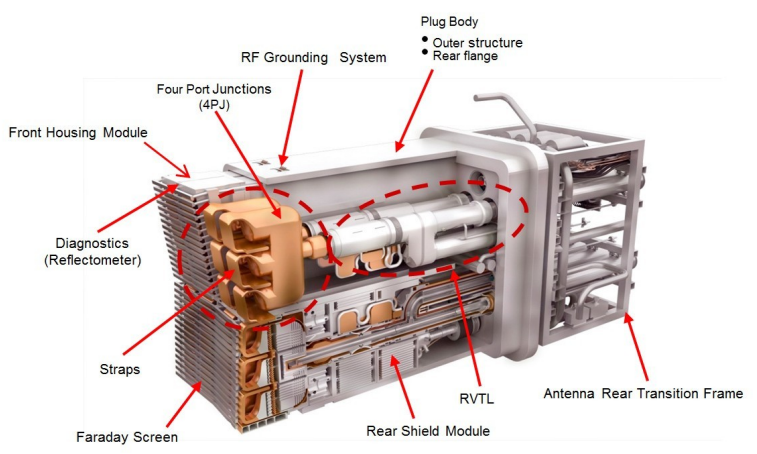

Figure 2: ITER ICRH antenna.

\section{FEM approach vs. transmission line approach}

Finite Element Modeling (FEM) codes with a plasma model as Topica [10] allow calculating the antenna input impedance and determine the distributions of electric and magnetic fields in the whole antenna with as input the plasma and Scrape-Off Layer (SOL) information (density and temperature profiles, composition), the static magnetic field information (amplitude and angle) and the antenna geometry. Plasma and SOL information can be used to create an equivalent dielectric load [11] if the FEM code doesn't have a plasma model, as it is the case for commercially available codes like CST MicroWave Studio [12] and ANSOFT HFSS [13]. The gyrotropy of the plasma is in this case not taken into account.

The FEM simulations indeed give a full RF characterization of the antenna but they require making use of very intensive computational resources. Hence the interest of the transmission line approach linked to a semi-analytical coupling code: it is very fast, requires limited computational resources and gives a very good approximation of the results. It also allows having a better understanding of the physical processes at hand.

\section{Strap input impedance}

As described in another lecture [2] the current strap can be described as a short-circuited strip-line with its distributed parameters $R_{A}, L_{A}, C_{A}$, which are determined by a semi-analytical coupling code, and length $l_{A}$. Using the $e^{+i \omega t}$ convention for the time dependence the strap input impedance, complex propagation constant and characteristic impedance are given by:

$$
\begin{gathered}
Z_{F}=R_{F}+i X_{F}=Z_{0 A} \tanh \left(\gamma_{A} l_{A}\right) \\
\gamma_{A}=\sqrt{\left(R_{A}+i \omega L_{A}\right) i \omega C_{A}} \\
Z_{0 A}=\sqrt{\frac{Z_{A}}{Y_{A}}}=\sqrt{\frac{R_{A}+i \omega L_{A}}{i \omega C_{A}}}
\end{gathered}
$$




\section{Choice of short straps}

The power radiated by the antenna is given by:

$$
P_{\text {rad }}=\int_{0}^{l_{A}} \frac{R_{A}|I(y)|^{2}}{2} d y,
$$

where $R_{A}$ is the radiation resistance as given by the coupling codes. If $R_{A}$ is determined experimentally one has to subtract the losses in the antenna (determined from tests on vacuum) from the experimentally measured loading resistance.

The current and voltage along the strap are given by:

$$
\begin{gathered}
I(y)=I_{\max } \cosh (\gamma y) \\
V(y)=Z_{0 A} I_{\max } \sinh (\gamma y)
\end{gathered}
$$

A comparison of the radiated power pattern, voltage and current along one long strap and three short straps is given in figure 3. As the radiated power is proportional to $|\cosh (\gamma y)|^{2}$ the power is very effectively radiated near the short circuit (where $y=0$ thus $\cosh (\gamma y) \approx 1$ ) and much less effectively when moving away from the short-circuit. The radiation efficiency is thus improved when going from one long strap to several short straps and for a given total power to be radiated the maximum current (at the short-circuit) will be lower. But the main advantage is the significant voltage reduction on the strap (about a factor 3 in the example of figure 3 ). The choice of short straps will lead to the design of high power density antennas.
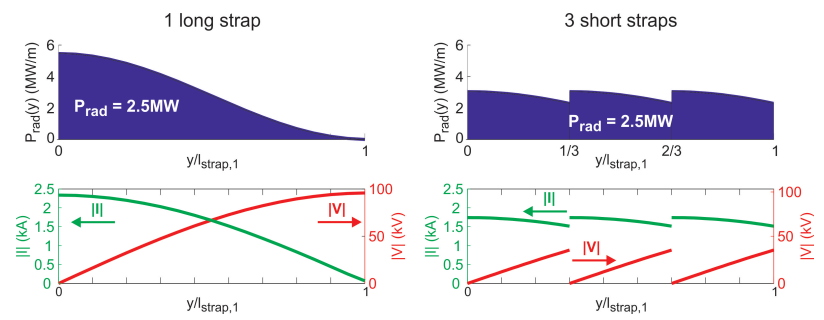

Figure 3: Radiated power pattern, voltage and current evolution along one long strap (left) and three shorter straps (right).

\section{Choice of the feeder characteristic impedance}

The main operating limit on current antennas is the maximum voltage in the system $V_{\max }$. We can express the transferred power by:

$$
P=\frac{G|V|^{2}}{2}=\frac{G_{\min }\left|V_{\max }\right|^{2}}{2}
$$

$G_{\min }$ is the minimum conductance, which will occur where $V=V_{\max }$ and represents the coupling to the plasma. The antenna optimization will consist in maximizing $G_{m i n}$, meaning maximizing the transferred power for a given allowed $V_{\max }$ on the system (or minimizing the maximum voltage on the system for a given power to transfer).

We have seen that by choosing short straps we could decrease the voltage on the straps. As the straps are shorter than a quarter wavelength $\left(l_{A}<\right.$ $\lambda / 4) V_{\max }$ will not occur on the strap and we need to include the next part, which is the strap feeder, into the system to be able to optimize the antenna.

The strap feeder is a cylindrical coaxial transmission line. The characteristic impedance of such a line with an inner radius $a$ and outer radius $b$ is:

$$
Z_{0}=\frac{1}{2 \pi} \sqrt{\frac{\mu}{\epsilon}} \ln \frac{b}{a} \approx 60 \ln \frac{b}{a}
$$

The maximum electric field will occur at the inner radius $a$ and is equal to:

$$
E_{\text {max }}=E(a)=\frac{V}{a \ln \left(\frac{b}{a}\right)}
$$

Figure 4 shows $G_{\min 1}$ and $E_{\max }$ in the feeder as a function of its characteristic impedance $\left(Z_{0 F}\right)$. To improve the performance of the system (i.e. maximize $\left.G_{\min 1}\right)$ one has to choose $Z_{0 F}$ as small as possible. But this reduces the gap between inner and outer radii of the feeder and $E_{\max }$ is dramatically increasing, finally exceeding the limit of $2 \mathrm{kV} / \mathrm{mm}$ (parallel to $B_{t}$ ), for small $Z_{0 F}$. The strap input impedance chosen to estimate the performance and electric field in figure 4 is taken from Topica calculations with reference ITER plasma and SOL profiles and for $0 \pi \pi 0$ toroidal phasing. $Z_{0 F}=15 \Omega$ is chosen as a good compromise between voltage stand-off and performance.

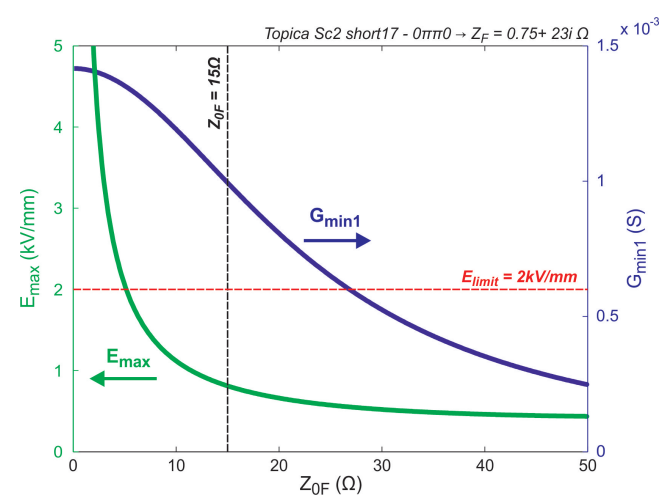

Figure 4: Minimum conductance $\left(G_{\min 1}\right)$ and maximum electric field $\left(E_{\max }\right)$ in the feeder in function of its characteristic impedance $\left(Z_{0 F}\right)$.

\section{Antenna segmentation}

Short straps were chosen to limit the voltage on the straps and have high power density. But why were three straps chosen as optimal for ITER ?

Let's consider the ITER antenna box and split it into $N$ segments. In the case of short straps - but not too short - the strap input impedance, mainly inductive, is roughly proportional to the length of the strap 
$\left(Z_{F, N} \approx Z_{F, 1} / N\right)$. The power of $2.5 M W$ per feeding line is divided amongst the $N$ segments. The feeder outer radius is constrained by the physical dimensions of the ITER antenna box $(320 \mathrm{~mm}$ wide, $945 \mathrm{~mm}$ high and $30 \mathrm{~mm}$ thick strap). $Z_{0 F}=15 \Omega$.

$V_{\max }, E_{\max }$ and $\left\langle P_{\text {loss }}\right\rangle$ on the feeder section are shown in figure 5 in function of the number of segments $N . E_{\max }$ and $\left\langle P_{\text {loss }}\right\rangle$ are increasing with $N$ but because of the constraints on the physical dimensions they also increase when going towards $N=1$ or 2. $V_{\max }$ is decreasing with $N$ as the power is divided among more straps. Again the choice is a matter of trade-off and $N=3$ is a good compromise between $E_{\max }$ and $V_{\max }$ on the feeder.

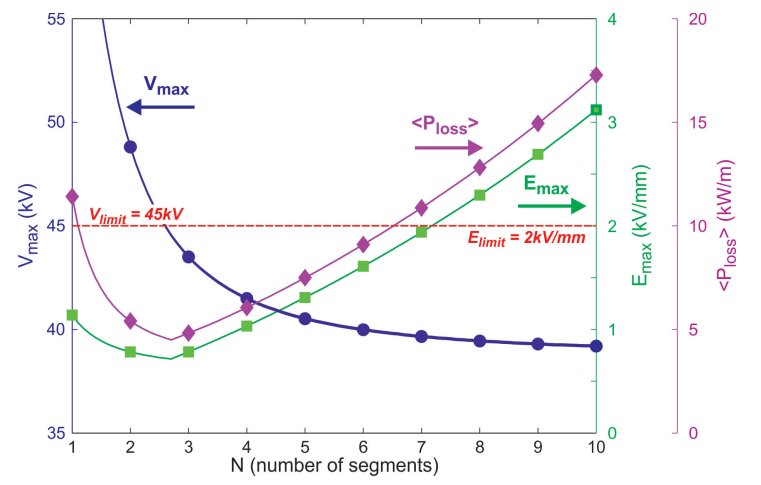

Figure 5: Maximum voltage $V_{\max }$, maximum electric field $E_{\text {max }}$ and average power loss $\left\langle P_{\text {loss }}\right\rangle$ in the feeder as a function of the segmentation of the antenna.

\section{Passive power distribution: the 4-port junction}

From the plasma point of view (or coupling) three short straps will be roughly equivalent to one long strap only if the currents flow in phase in the three straps. This can be achieved either actively or passively. An active control requires a large number of matching components to ensure the correct phase on the three straps, which in turn will lead to space issues and a very complex matching (the 24 straps are mutually coupled). This leads to the choice of a passive power distribution by the use of a multi-port junction: 3 straps are connected in parallel by a 4 -port junction (4PJ). In this case the currents will be in phase if the 4-port junction is located around $V_{\max }$.

This has consequences on the frequency response of the antenna. Let's note $G_{\min 1}$ the minimum conductance in the strap feeder section (antenna side of the $4 \mathrm{PJ}$ ) and $G_{\min 2}$ the minimum conductance in the feeding line of one strap triplet (generator side of the $4 \mathrm{PJ}$ ). For a given antenna geometry and loading conditions $G_{\min 1}$ is evaluated by a coupling code. If the junction is located at the voltage anti-node $\left(V_{\max }\right)$ for all frequencies then $G_{\min 2}=G_{\min 2, \max }=3 G_{\min 1}$. But in practice the junction is physically located at a fixed position and will be at $V_{\max }$ for a single frequency. Moving away from this point in frequency will lead to a $G_{\min 2}<G_{\min 2, \max }$ (see figure 6 ). The 4-port junction acts as a frequency filter. The position of the maximum of $G_{m i n 2}$ can be moved towards lower/higher frequency by increasing/reducing the feeder or 4PJ length.

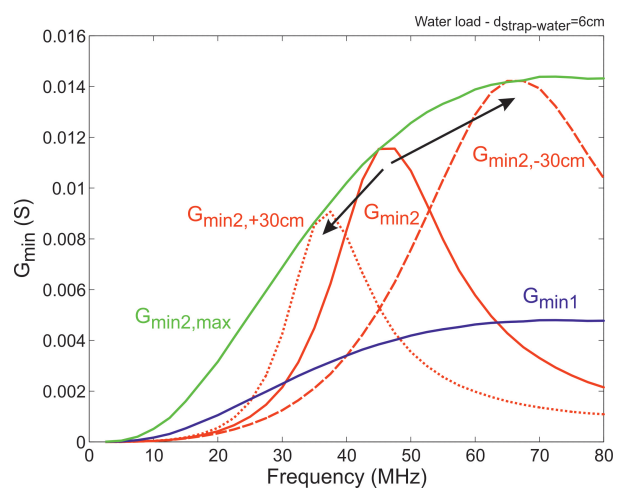

Figure 6: $G_{\min 1}, G_{\min 2, \max }$ and $G_{\min 2}$ (to have $V_{\max }$ at mid-band) vs. frequency. $G_{\min 2,-30 \mathrm{~cm}}$ and $G_{m i n 2,+30 \mathrm{~cm}}$ when removing or adding $30 \mathrm{~cm}$ of feeder length.

\section{Antenna optimization [14, 15]}

The main operating limit on current antennas being $V_{\max }$ the RF antenna design will strive to:

- improve the achievable $V_{\max }$ by design;

- maximize $G_{\min 2}$, i.e. maximize the transferred power for a given $V_{\max }$.

Increasing $G_{\min 2}$ can be obtained by increasing $G_{m i n 1}$, i.e. by acting on the antenna front face geometry (strap width, box depth, septum recess). As the strap input impedance is mostly reactive $\left(R_{F}^{2}<<\right.$ $X_{F}^{2}$ ) we have:

$$
G_{m i n 1} \approx \frac{R_{F}}{X_{F}^{2}+Z_{0 F}^{2}}
$$

The resistive part, $R_{F}$, is determined partly by the antenna box geometry and partly by the external medium (mainly plasma and SOL density profiles). The reactive term, $X_{F}$, depends mainly on the antenna box geometry and only weakly depends on the external medium.

Maximizing $G_{\min 1}$ often leads to a reduction of $X_{F}$ accompanied by a reduction in $R_{F}$. The current in the antenna then increases as $\left|I_{F}\right|=\left|V_{F}\right| /\left|Z_{F}\right| \approx$ $\left|V_{F}\right| /\left|X_{F}\right|$. The ITER ICRF antenna being a long pulse antenna it needs active cooling. This sets a limit on the maximum allowed RF losses, thus the maximum allowed current (a.o. in the RF contacts). The RF optimization has to proceed together with the thermo-mechanical calculations in order to find the best trade-off between the limits on maximum current, electric field and voltage.

$G_{\min 2}$ can also be maximized by the choice of the 4PJ geometry, which response should be as close as possible to the one of an ideal transmission line [14].

Finally it was shown [14] that broad-banding of the system response in the main transmission line 
could be obtained by introduction of a quarter-wave stub at a quarter-wavelength of the 4PJ.

\section{MATCHING}

The matching has three essential goals:

- maximizing the power transfer to the antenna and minimizing the power loss in the feed line;

- controlling the antenna array current spectrum;

- avoiding tripping the generator.

When a generator is delivering its power through a transmission line to a load the maximum power transfer occurs when the input impedance looking into the transmission line terminated by the load from the generator end is the complex conjugate of the output impedance of the generator [6].

In practice, the generator output impedance is real and equal to $Z_{0 G}=30 \Omega$ or $50 \Omega$. The strap input impedance is complex and essentially reactive. To maximize the transferred power we need to transform $Z_{F}$ into $Z_{0 G}$. This is the first role of the matching circuit.

Secondly the different heating and current drive scenarios require different antenna array current spectra: the phase and amplitude of the currents of the different poloidal columns of straps need to be controlled.

Finally the generators are protected against too high power reflection to avoid any damage (they are typically limited to $V S W R<1.5$, i.e. $\left.\left|\Gamma_{G}\right|<0.2\right)$. Above this limit the generator is tripped (no power is delivered any more).

\section{A. Classical Matching}

The principle of classical matching is shown in figure 7. A generator delivers incident power $\left(P_{i n}\right)$ to the system. Its output impedance is real (e.g. $Z_{0 G}=50 \Omega$ ). The antenna has a complex input impedance $Z_{F}=R_{F}+i X_{F}$, with $R_{F}^{2}<<X_{F}^{2}$. The transmission line is generally matched to the generator $\left(Z_{0 T L}=Z_{0 G}\right)$. Because of the impedance mismatch $\left(Z_{F} \neq Z_{0 T L}\right)$ most of the delivered power is reflected at the antenna and can cause tripping of the generator. To fully radiate $P_{i n}$ a matching circuit is inserted to constitute a resonant circuit, in which VSWR is high, with the antenna. It increases the antenna current to the value needed to radiate $P_{\text {in }}$ and low (no in case of perfect match) power is reflected back to the generator. The generator side of the matching circuit is a matched section where $V S W R \approx 1$. The load of the antenna is not constant: varying plasma and SOL conditions (confinement regime transition, distance plasma-antenna, switching on of NBI) lead to changes in the strap input impedance: mainly in the resistive part $\left(R_{F}\right)$ but also to a lesser extent in the reactive part $\left(X_{F}\right)$. Consequently the elements constituting the matching circuit need to be adjustable. The most widely used matching components are:

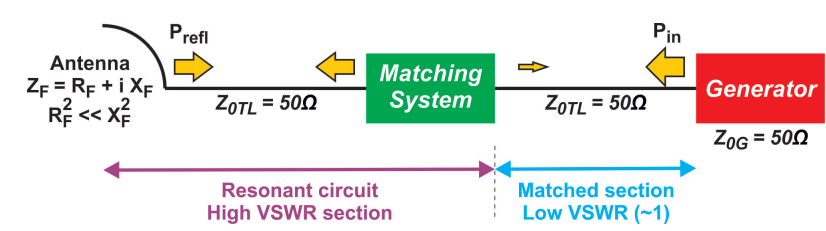

Figure 7: Principle of classical matching.

- reactive components such as capacitors or shunt short-circuited line sections called stubs;

- phase shifters or line stretchers.

The admittance of a capacitor is purely capacitive: $Y=i X$, with $X=\omega C$.

The impedance of a lossless short-circuited line section (or stub) is: $Z_{s t}=i Z_{0} \tan \left(\beta l_{S t}\right)$. The stub can be either capacitive or inductive depending on its length $l_{S t}$.

The phase shifter is a section of transmission line of variable electrical length: it changes the phase angle of the reflection coefficient. This length can be changed mechanically by using trombones or electrically as on JET by a slight frequency shift on very long lines [16].

Other techniques are employed as modifying the electrical properties of the transmission line (e.g. liquid tuners [17]) or its magnetic properties (e.g. ferrite tuners [18]).

\section{The single-stub tuner}

The single stub tuner is a matching circuit composed of a line stretcher and a shunt stub, whose lengths are the two adjustable parameters.

The length of the line stretcher is chosen such that the input admittance looking towards the load at the stub insertion position is of the form $Y=Y_{0 G}+$ $i B$ (where $Y_{0 G}=1 / Z_{0 G}$ ). The length of the stub is then chosen such that its susceptance is equal to $-i B$ resulting in a matched condition.

There are two solutions: one inductive and one capacitive. All loads can be matched provided the available lengths are sufficient.

\section{The double-stub tuner}

This matching circuit is composed of two shunt stubs, whose lengths constitute the two adjustable parameters, distant by typically $\lambda / 8$ for the mid-band frequency.

The first stub (antenna side) is set such that the input admittance looking towards the load at the position of the insertion of the second stub (generator side) is of the form $Y=Y_{0 G}+i B$. Again the length of the second stub is chosen to neutralize the remaining susceptance.

This scheme doesn't allow matching all loads: there's an inaccessible region that depends on the distance between the two stubs. Adding a line stretcher however allows to move outside the inaccessible region. 


\section{B. ELM-resilient matching [19, 20]}

The classical matching components are too slow to cope with the very fast load changes due to ELMs (Edge Localized Modes; they appeared with the discovery of the H-mode). The resistive load during ELMs is typically increased by a factor 2 to 4 while the inductive load typically decreases less dramatically by about 2 to $15 \%$.

Two ELM-resilient schemes were developed and tested on several machines: the Conjugate-T (TEXTOR [21], Tore-Supra [22], JET [23, 24]), which is presently the ITER back-up option, and the hybrid coupler [25] (DIII-D [26], ASDEX-U [27], JET [28]), which is presently the ITER reference one [8].

\section{The Conjugate- $T$}

In this scheme two straps (two poloidal triplets for the ITER ICRH antenna) are connected to a single feeding line by a T-junction (see figure 8). A reactive element - generally a capacitor or a shunt stub - is added in each line between the strap and the T-junction. Its susceptance is chosen such that one branch is inductive with an admittance at the $\mathrm{T}$ junction equal to $Y_{1}=1 /(R+i|X|)$ and the other is capacitive with the complex conjugate admittance of the first branch at the T-junction: $Y_{2}=Y_{1}^{*}=$ $1 /(R-i|X|)$. The admittance in the feeding line at the T-junction is then $Y_{T}=Y_{1}+Y_{1}^{*}=1 / Z_{0 G}$.

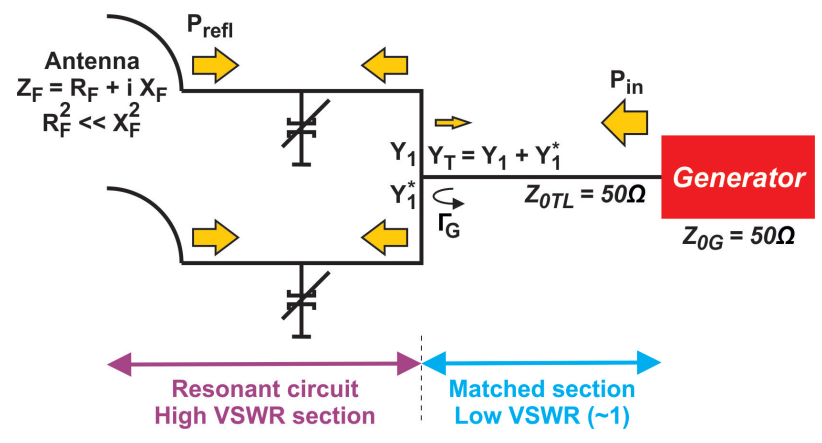

Figure 8: Conjugate-T circuit.

If only the resistive part of the load varies during an ELM then we can express the reflection coefficient at the T-junction towards the generator in function of the normalized variables $r=R / Z_{0 G}$ and $x=X / Z_{0 G}$ as:

$$
\Gamma_{G}=\frac{\left(r-r_{1}\right)\left(r-r_{2}\right)}{\left(r+r_{1}\right)\left(r+r_{2}\right)}
$$

with $r_{1} r_{2}=x^{2}$ and $r_{1}+r_{2}=2$.

The evolution of $\left|\Gamma_{G}\right|$ in function of $\mathrm{r}$ is shown on figure 9. Perfect match occurs for $r=r_{1}$ and $r=r_{2}$. For $r 1<r<r 2$ the curve presents a maximum at $r=r_{s}$ corresponding to a VSWR of $S=S_{\max }=1 / x$. We can see that there is a large load-resilient domain $\left(\left[r_{b 1}, r_{b 2}\right]\right)$, where $S<S_{\max }$.

The price to pay for the load resilience is a change in phase angle between the currents flowing in the two branches, given by $\Delta \Phi=2 \arctan \left(\sqrt{r_{1} r_{2}} / r\right)$.
A second stage matching circuit (or impedance transformer) can be implanted between the generator and the T-junction in order to change the impedance on which one has to match at the T-junction. In this case one can improve the load resilience by lowering the impedance to match at the T-junction. This is especially useful for low loadings where the matching is more difficult to achieve. This also allows imposing a complex impedance on which to match at the Tjunction, what can be useful in case of strong mutual coupling between different Conjugate- $\mathrm{T}$ circuits.

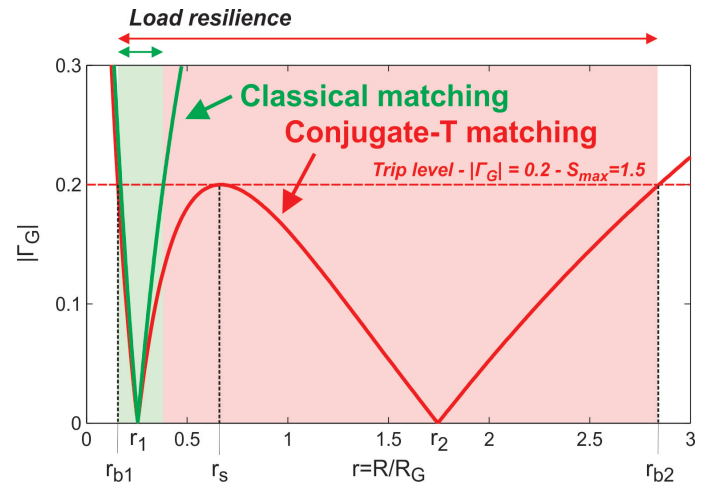

Figure 9: Reflection coefficient vs. normalized loading resistance for CT matching and classical matching. Load resilience domain is much larger for CT matching.

The Conjugate-T matching requires symmetric $R$ and $X$ variations and has limited resilience to $X$ variations.

One of its most remarkable features is that it continues to deliver the power through the ELMs. The debate on whether this is an advantage or a drawback (it could favor arcing in this phase where plasma is expelled towards the antenna) is still open.

\section{The Hybrid Coupler}

In this case the power is delivered through a hybrid coupler to two straps (two poloidal triplet of straps for the ITER ICRH antenna). The last port of the hybrid coupler is connected to a dummy load (see figure 10).

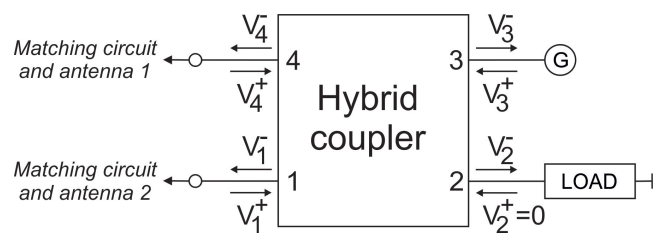

Figure 10: Hybrid coupler circuit.

The scattering matrix of the hybrid coupler is:

$$
S=-\left(\begin{array}{cccc}
0 & i \delta & \alpha & 0 \\
i \delta & 0 & 0 & \alpha \\
\alpha & 0 & 0 & i \delta \\
0 & \alpha & i \delta & 0
\end{array}\right) \text {, where } V_{i}^{-}=\sum_{j} S_{i j} V_{j}^{+}
$$


For the $3 \mathrm{~dB}$ hybrid coupler $\alpha=\delta=1 / \sqrt{2}$ giving:

$$
V_{1}^{-}=\frac{-1}{\sqrt{2}} V_{3}^{+} ; V_{4}^{-}=\frac{-i}{\sqrt{2}} V_{3}^{+}
$$

In this case the power is split into two equal parts to the two branches but with a phase difference of $\pi / 2$.

The matching is done by a classical matching circuit placed between the antenna current straps and the $3 \mathrm{~dB}$ hybrid coupler. For perfectly matched conditions, no power is reflected back to the hybrid coupler. But when an ELM occurs the system is not matched any more and a high fraction of power is reflected towards the hybrid.

The reflection to the generator and transfer coefficient to the dummy load are given by:

$$
\Gamma_{3}=\frac{V_{3}^{-}}{V_{3}^{+}}=\frac{\Gamma_{1}-\Gamma_{4}}{2} ; T_{L G}=\frac{V_{2}^{-}}{V_{3}^{+}}=i \frac{\Gamma_{1}+\Gamma_{4}}{2}
$$

No reflection to the generator and full dump of the reflected power will happen when $\Gamma_{1}$ and $\Gamma_{4}$ are equal in phase and amplitude. This needs complete symmetry in the load variation $(R$ and $X)$ and of the matching circuit.

In the $3 \mathrm{~dB}$ hybrid coupler scheme the generator continues to deliver power during the ELMs but most of this power is dumped into the dummy load. The generator is isolated from the load changes.

\section{Decouplers}

The ELM-resilient schemes not only require that the load variations on the two branches are symmetric but also that the level of mutual coupling is kept sufficiently low.

This last condition pleads in favor of the use of decouplers when possible (it is difficult to implement them with internal matching for instance).

A decoupler consists of two $\lambda / 4$ lines of characteristic impedance $Z_{0 \text { dec }}$ connected to an adjustable reactance $X_{d e c}$ (see figure 11) [20].

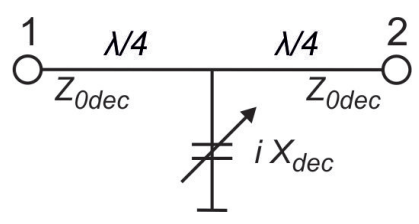

Figure 11: Decoupler circuit.

All the terms of the $2 \times 2$ admittance matrix $Y_{d e c}$ of this two-port network are equal to $i X_{d e c} / Z_{0 d e c}^{2}$. This circuit connected in parallel to a two-port network with admittance $Y$ allows to cancel the reactive part of the coupling terms if $Y_{12}=Y_{21}$ or to reduce it in case of a non-symmetric matrix $\left(Y_{12} \neq Y_{21}\right)$, which is the case on plasma. The decoupler adds an additional contribution to the diagonal terms $\left(Y_{11}\right.$ and $Y_{22}$ ) that has to be taken care of by the matching circuit.

\section{REFERENCES}

1. E. Lerche and D. Van Eester, Kinetic theory of plasma waves, These Proc.

2. F. Louche and R. Koch, The coupling of electromagnetic power to plasmas, These Proc.

3. R. Koch, The ion cyclotron, lower hybrid and Alfvén wave heating methods, These Proc.

4. E. Westerhof, Current drive, These Proc.

5. M.I.T. Radiation Laboratory Series: 28 Volumes on 2 CD-Roms, ARTECH House (1999)

6. D.M. Pozar, Microwave Engineering, John Wiley ES Sons, Inc. (2004)

7. F. Durodié, Transactions of Fusion Technology, 25(2T):Part 2, 284-288 (1994)

8. P. Lamalle et al., Fusion Engineering and Design, In Press, doi:10.1016/j.fusengdes.2012.11.027 (2013)

9. D. Hancock et al.,AIP Conference Proceedings, 1406, 57-60 (2011)

10. V. Lancellotti et al., Nucl. Fusion, 46, S476 (2006).

11. A. Messiaen et al., Fusion Engineering and Design, 86, 855-859 (2011)

12. CST GmbH, CST Microwave Studio User Manual (2009), http://www.cst.com

13. "High Frequency Structure Simulator (TM)", Ansoft Corp., http://www.ansoft.com

14. P. Dumortier et al., Fusion Engineering and Design, 84, 707711 (2009)

15. F. Durodié et al., "Performance Assessment of the ITER ICRF Antenna", Proc. of the 24th IAEA Fusion Energy Conference (2012)

16. T.J. Wade et al., Fusion Engineering and Design, 24, 23-46 (1994)

17. R. Kumazawa et al., AIP Conference Proceedings, 485, 441-444 (1999)

18. S. Martin et al., AIP Conference Proceedings, 244, 318-321 (1992)

19. J.M. Noterdaeme et al., Fusion Engineering and Design, 74, 191-198 (2005)

20. A. Messiaen et al., Nuclear Fusion, 49, 055004 (2009)

21. F. Durodié et al, Fusion Engineering and Design, 66-68, 509-513 (2003)

22. K. Vulliez et al., Fusion Engineering and Design, 66-68, 531-535 (2003)

23. F. Durodié et al., AIP Conference Proceedings, 595, 122-125 (2001)

24. F. Durodié et al., Fusion Engineering and Design, 84, 279-283 (2009)

25. R. Goulding et al., AIP Conference Proceedings, 355, 397-400 (1995)

26. R.I. Pinsker et al., Plasma Physics and Controlled Fusion, 40, A215-A229 (1998)

27. J.M. Noterdaeme et al., 16th IAEA Conf. on Fusion Energy, 3, 335-342 (1996)

28. M. Vrancken et al., Fusion Engineering and Design, 82, 873-880 (2007) 